\title{
On A Generalized Köthe-Toeplitz Duals
}

\author{
Sumardyono ${ }^{1}$, Supama $^{2}$, dan Soeparna Darmawijaya ${ }^{3}$ \\ ${ }^{1}$ PPPPTK Matematika, smrdyn2007@gmail.com \\ ${ }^{2}$ Mathematics Department, Gadjah Mada University, supama@ugm.ac.id \\ ${ }^{3}$ Mathematics Department, Gadjah Mada University
}

\begin{abstract}
In this paper, we generalized the notion of Kothe-Toeplitz duals of sequence space on introducing the concept sequence of sequences. Some properties of the generalized dual are obtained.
\end{abstract}

Keywords: Köthe-Toeplitz duals, Generalized dual spaces, Sequence of sequences

ABSTRAK. Pada paper ini dibahas mengenai dual Kothe-Toeplitz yang diperumum dari ruang barisan ke ruang barisan dengan suku barisan. Selain itu, akan dibahas juga mengenai sifat-sifat dari dual tersebut.

Kata Kunci: dual Kothe-Toeplitz, ruang dual yang diperumum, barisan dari barisan

\section{PENDAHULUAN}

Konsep ruang dual merupakan konsep penting dalam studi analisis. Salah satu bahasan mengenai dualitas suatu ruang telah disampaikan Köthe dan Toeplitz (1934), Garling (1967), dan juga Ruckle (1967) dengan memperkenalkan tipe-tipe dual ruang barisan yaitu dual- $\alpha$, dual- $\beta$, dual- $\gamma$, dan dual- $\delta$. Sifat-sifat dual- $\alpha$, dual- $\beta$, dan dual- $\gamma$ di atas juga telah dibahas oleh Kamthan \& Gupta.

Berangkat dari pengertian di atas, kami memperluas konsep dual- $\alpha$, dual$\beta$, dual- $\gamma$, dan dual- $\delta$ dari ruang barisan ke ruang barisan dengan suku barisan.

\section{PERLUASAN DUAL KÖTHE-TOEPLITZ}

Dimisalkan $\omega$ koleksi semua barisan $x=\left\{x_{n}\right\}$ dengan $x_{n} \in \mathrm{C}, n \geq 1$. 
Hasil kali dan penjumlahan dua barisan $\left\{x_{n}\right\}$ dan $\left\{y_{n}\right\}$ didefinisikan secara pointwise sebagai berikut.

$$
\begin{aligned}
\left\{x_{n}\right\} .\left\{y_{n}\right\} & =\left\{x_{1}, x_{2}, x_{3}, \ldots\right\} .\left\{y_{1}, y_{2}, y_{3}, \ldots\right\} \\
& =\left\{x_{1} . y_{1}, x_{2} . y_{2}, x_{3} . y_{3}, \ldots\right\} \\
\left\{x_{n}\right\}+\left\{y_{n}\right\} & =\left\{x_{1}, x_{2}, x_{3}, \ldots\right\}+\left\{y_{1}, y_{2}, y_{3}, \ldots\right\} \\
& =\left\{x_{1}+y_{1}, x_{2}+y_{2}, x_{3}+y_{3}, \ldots\right\}
\end{aligned}
$$

Dengan penjumlahan pointwise dan perkalian skalar, maka $\omega$ menjadi ruang vektor atas $\mathrm{C}$.

Himpunan semua permutasi dari $\mathrm{N}$ dinotasikan dengan $\pi$.

Definisi 2.1 ([6], [1], [5]) Dipandang ruang vektor tak nol $\lambda \subset \omega$ maka didefinisikan dual- $\alpha$, dual- $\beta$, dual $-\gamma$, dan dual- $\delta$ dari $\lambda$ berturut-turut sebagai berikut.

$$
\begin{aligned}
& \lambda^{\alpha}=\left\{x: x \in \omega, \sum_{i \geq 1}\left|x_{i} y_{i}\right|<\infty, \forall y \in \lambda\right\} \\
& \lambda^{\beta}=\left\{x: x \in \omega,\left|\sum_{i \geq 1} x_{i} y_{i}\right|<\infty, \forall y \in \lambda\right\} \\
& \lambda^{\gamma}=\left\{x: x \in \omega, \sup _{n}\left|\sum_{i=1}^{n} x_{i} y_{i}\right|<\infty, \forall y \in \lambda\right\} \\
& \lambda^{\delta}=\left\{x: x \in \omega, \sum_{i \geq 1}\left|x_{i} y_{\rho(i)}\right|<\infty, \forall y \in \lambda, \text { dan } \rho \in \pi\right\}
\end{aligned}
$$

Selanjutnya, untuk setiap $x=\left\{x_{n}\right\} \in \omega$, beberapa notasi didefinisikan sebagai berikut.

$$
|x|_{1}=\left|\left\{x_{n}\right\}\right|_{1}=\left|x_{1}\right|+\left|x_{2}\right|+\left|x_{3}\right|+\ldots \text { dan }|x|=\left|\left\{x_{n}\right\}\right|=\left\{\left|x_{1}\right|,\left|x_{2}\right|,\left|x_{3}\right|, \ldots\right\} .
$$

Sehingga $|x|_{1} \in \mathrm{C}$ sedang $|x| \in \omega$.

Barisan dengan entri di $\omega$ dinotasikan dengan huruf kapital $X=\left\{x^{(n)}\right\}$ dengan $x^{(n)}$ menyatakan suku ke- $n$ pada $X$.

$$
\begin{aligned}
& X=\left\{x^{(1)}, x^{(2)}, x^{(3)}, \ldots\right\} \\
& \text { dengan } \quad x^{(1)}=\left\{x_{11}, x_{12}, x_{13}, \ldots\right\} \in \omega
\end{aligned}
$$




$$
\begin{aligned}
& x^{(2)}=\left\{x_{21}, x_{22}, x_{23}, \ldots\right\} \in \omega \\
& x^{(3)}=\left\{x_{31}, x_{32}, x_{33}, \ldots\right\} \in \omega
\end{aligned}
$$

Dimisalkan $\Omega$ koleksi semua barisan $X=\left\{x^{(n)}\right\}$ dengan $x^{(n)} \in \omega$. Hasil kali dan penjumlahan pada $\Omega$ dilakukan secara pointwise sebagai berikut.

Untuk setiap $X, Y \in \Omega$ maka

$$
\begin{aligned}
X . Y & =\left\{x^{(n)}\right\} .\left\{y^{(n)}\right\}=\left\{x^{(1)} \cdot y^{(1)}, x^{(2)} \cdot y^{(2)}, x^{(3)} \cdot y^{(3)}, \ldots .\right\} \\
X+Y & =\left\{x^{(n)}\right\}+\left\{y^{(n)}\right\}=\left\{x^{(1)}+y^{(1)}, x^{(2)}+y^{(2)}, x^{(3)}+y^{(3)}, \ldots .\right\}
\end{aligned}
$$

Dengan penjumlahan bersifat pointwise dan perkalian skalar, maka $\Omega$ menjadi sebuah ruang vektor atas $\mathrm{C}$.

Definisi 2.2 Dipandang $\Lambda \subset \Omega$ sebuah ruang vektor atau ruang linear yang tak nol. Dengan $X=\left\{x^{(n)}\right\}$ dan $Y=\left\{y^{(n)}\right\}, x^{(n)}, y^{(n)} \in \omega$ maka didefinisikan berturutturut,

$$
\begin{aligned}
& \Lambda^{\alpha}=\left\{X: X \in \Omega, \sum_{i \geq 1}\left|x^{(i)} y^{(i)}\right|_{1}<\infty, \forall Y \in \Lambda\right\} \\
& \Lambda^{\beta}=\left\{X: X \in \Omega,\left|\sum_{i \geq 1} x^{(i)} y^{(i)}\right|_{1}<\infty, \forall Y \in \Lambda\right\} \\
& \Lambda^{\gamma}=\left\{X: X \in \Omega, \sup _{n}\left|\sum_{i=1}^{n} x^{(i)} y^{(i)}\right|_{1}<\infty, \forall Y \in \Lambda\right\} \\
& \Lambda^{\delta}=\left\{X: X \in \Omega, \quad \sum_{i \geq 1}\left|x^{(i)} y^{\rho(i)}\right|_{1}<\infty, \forall Y \in \Lambda \text { dan } \rho \in \pi\right\}
\end{aligned}
$$

Selanjutnya, berturut-turut ruang $\Lambda^{\alpha}, \Lambda^{\beta}, \Lambda^{\gamma}$, dan $\Lambda^{\delta}$ disebut dual- $\alpha$, dual- $\beta$, dual- $\gamma$, dan dual- $\delta$ dari $\Lambda$.

Dengan mengingat bahwa untuk setiap $x=\left\{x_{n}\right\} \in \omega$, bentuk $|x|_{1}$ didefinisikan sebagai $\left|x_{1}\right|+\left|x_{2}\right|+\left|x_{3}\right|+\ldots$ maka diperoleh sebagai berikut.

$$
\sum_{i \geq 1}\left|x^{(i)} y^{(i)}\right|_{1}=\left|x^{(1)} y^{(1)}\right|_{1}+\left|x^{(2)} y^{(2)}\right|_{1}+\left|x^{(3)} y^{(3)}\right|_{1}+\ldots
$$




$$
\begin{aligned}
= & \left|\left\{x_{11}, x_{12}, x_{13}, \ldots\right\} .\left\{y_{11}, y_{12}, y_{13}, \ldots\right\}\right|_{1}+ \\
& \left|\left\{x_{21}, x_{22}, x_{23}, \ldots\right\} \cdot\left\{y_{21}, y_{22}, y_{23}, \ldots\right\}\right|_{1}+ \\
& \left|\left\{x_{31}, x_{32}, x_{33}, \ldots\right\} \cdot\left\{y_{31}, y_{32}, y_{33}, \ldots\right\}\right|_{1}+\ldots \\
= & \left|\left\{x_{11} \cdot y_{11}, x_{12} \cdot y_{12}, x_{13} \cdot y_{13}, \ldots\right\}\right|_{1}+\left|\left\{x_{21} \cdot y_{21}, x_{22} \cdot y_{22}, x_{23} \cdot y_{23}, \ldots\right\}\right|_{1}+ \\
& \left|\left\{x_{31} \cdot y_{31}, x_{32} \cdot y_{32}, x_{33} \cdot y_{33}, \ldots\right\}\right|_{1}+\ldots \\
= & \left\{\left|x_{11} y_{11}\right|+\left|x_{12} y_{12}\right|+\left|x_{13} y_{13}\right|+\ldots\right\}+\left\{\left|x_{21} y_{21}\right|+\left|x_{22} y_{22}\right|+\right. \\
& \left.\left|x_{23} y_{23}\right|+\ldots\right\}+ \\
& \left\{\left|x_{31} y_{31}\right|+\left|x_{32} y_{32}\right|+\left|x_{33} y_{33}\right|+\ldots\right\}+\ldots \\
= & \sum_{j \geq 1}\left|x_{1 j} y_{1 j}\right|+\sum_{j \geq 1}\left|x_{2 j} y_{2 j}\right|+\sum_{j \geq 1}\left|x_{3 j} y_{3 j}\right|+\ldots \\
= & \sum_{i \geq 1} \sum_{j \geq 1}\left|x_{i j} y_{i j}\right|
\end{aligned}
$$

Selanjutnya dengan cara yang sama diperoleh berturut-turut.

$$
\begin{aligned}
& \left|\sum_{i \geq 1} x^{(i)} y^{(i)}\right|_{1}=\sum_{j \geq 1}\left|\sum_{i \geq 1} x_{i j} y_{i j}\right| \\
& \sup _{n}\left|\sum_{i=1}^{n} x^{(i)} y^{(i)}\right|_{1}=\sup _{n} \sum_{j \geq 1}\left|\sum_{i=1}^{n} x_{i j} y_{i j}\right| \\
& \sum_{i \geq 1}\left|x^{(i)} y^{\rho(i)}\right|_{1}=\sum_{i \geq 1} \sum_{j \geq 1}\left|x_{i j} y_{\rho(i) j}\right|
\end{aligned}
$$

Dengan demikian, dual- $\alpha$, dual- $\beta$, dual- $\gamma$, dan dual- $\delta$ dari $\Lambda$ dapat dinyatakan kembali sebagai berikut.

Definisi 2.3 Dipandang $\Lambda \subset \Omega$ sebuah ruang vektor atau ruang linear yang tak nol. Dengan $X=\left\{x^{(n)}\right\}$ dan $Y=\left\{y^{(n)}\right\}, x^{(n)}, y^{(n)} \in \omega$ serta $x^{(i)}=\left\{x_{i j}\right\}$ dan $y^{(i)}=\left\{y_{i j}\right\}$ maka didefinisikan berturut-turut,

$$
\begin{aligned}
& \Lambda^{\alpha}=\left\{X: X \in \Omega, \sum_{i \geq 1} \sum_{j \geq 1}\left|x_{i j} y_{i j}\right|<\infty, \forall Y \in \Lambda\right\} \\
& \Lambda^{\beta}=\left\{X: X \in \Omega, \sum_{j \geq 1}\left|\sum_{i \geq 1} x_{i j} y_{i j}\right|<\infty, \forall Y \in \Lambda\right\} \\
& \Lambda^{\gamma}=\left\{X: X \in \Omega, \sup _{n} \sum_{j \geq 1}\left|\sum_{i=1}^{n} x_{i j} y_{i j}\right|<\infty, \forall Y \in \Lambda\right\}
\end{aligned}
$$




$$
\Lambda^{\delta}=\left\{X: X \in \Omega, \quad \sum_{i \geq 1} \sum_{j \geq 1}\left|x_{i j} y_{\rho(i) j}\right|<\infty, \forall Y \in \Lambda \text { dan } \rho \in \pi\right\}
$$

Pandang koleksi $\Omega^{*}=\left\{X \in \Omega: X=\left\{x^{(n)}\right\}, x^{n}=\left\{x_{n}, 0,0,0, \ldots\right\}, n \geq 1\right\}$ dan $\Lambda \subset$ $\Omega^{*}$ ruang vektor tak nol, maka

$$
\begin{array}{ll}
\sum_{i \geq 1} \sum_{j \geq 1}\left|x_{i j} y_{i j}\right|=\sum_{i \geq 1}\left|x_{i} y_{i}\right|, & \sum_{j \geq 1}\left|\sum_{i \geq 1} x_{i j} y_{i j}\right|=\left|\sum_{i \geq 1} x_{i} y_{i}\right| \\
\sup _{n} \sum_{j \geq 1}\left|\sum_{i=1}^{n} x_{i j} y_{i j}\right|=\sup _{n}\left|\sum_{i=1}^{n} x_{i} y_{i}\right| & \sum_{i \geq 1} \sum_{j \geq 1}\left|x_{i j} y_{\rho(i) j}\right|=\sum_{i \geq 1}\left|x_{i} y_{\rho(i)}\right|
\end{array}
$$

Diperoleh bahwa.

$$
\begin{aligned}
& \Lambda^{\alpha}=\lambda^{\alpha}, \quad \Lambda^{\beta}=\lambda^{\beta}, \quad \Lambda^{\gamma}=\lambda^{\gamma}, \quad \Lambda^{\delta}=\lambda^{\alpha} \\
& \text { dengan } \lambda=\left\{\left\{y_{j}\right\}: y_{j} \in \mathrm{C}\right\} \subset \omega, \text { dan } \omega=\left\{\left\{x_{j}\right\}: x_{j} \in \mathrm{C}\right\}
\end{aligned}
$$

Jadi $\Lambda^{\alpha}, \Lambda^{\beta}, \Lambda^{\gamma}$, dan $\Lambda^{\delta}$ merupakan suatu perluasan atau generalisasi dari $\lambda^{\alpha}, \lambda^{\beta}$ ,$\lambda \gamma$, dan $\lambda^{\delta}$.

Sementara, untuk koleksi $\Omega^{* *}=\left\{X \in \Omega: X=\left\{x^{(1)}, 0,0,0, \ldots\right\}\right\}$ dan $\Lambda \subset \Omega^{* *}$ ruang vektor tak nol, maka

$$
\begin{aligned}
& \quad \Lambda^{\alpha}=\Lambda^{\beta}=\Lambda^{\gamma}=\Lambda^{\delta}=\lambda^{\alpha} \quad \text { dengan } \lambda=\left\{\left\{y_{j}\right\}: y_{j} \in \mathrm{C}\right\} \subset \omega \text {, dan } \omega=\left\{\left\{x_{j}\right\}\right. \\
& \left.: x_{j} \in \mathrm{C}\right\}
\end{aligned}
$$

\section{BEBERAPA SIFAT PERLUASAN DUAL KÖTHE-TOEPLITZ}

Pada bagian akhir ini, kami sampaikan beberapa sifat Perluasan dual KötheToeplitz yang masih serupa dengan sifat-sifat pada dual Köthe-Toeplitz.

Proposisi $3.1 \Lambda^{\alpha} \subset \Lambda^{\beta}$

Bukti: Diambil sebarang $X \in \Lambda^{\alpha}$ maka untuk sebarang $Y \in \Lambda$ diperoleh

$$
\begin{aligned}
\sum_{j \geq 1}\left|\sum_{i \geq 1} x_{i j} y_{i j}\right| & =\sum_{j \geq 1}\left|x_{1 j} y_{1 j}+x_{2 j} y_{2 j}+x_{3 j} y_{3 j}+\ldots\right| \\
\leq & \sum_{j \geq 1}\left\{\left|x_{1 j} y_{1 j}\right|+\left|x_{2 j} y_{2 j}\right|+\left|x_{3 j} y_{3 j}\right|+\ldots\right\} \\
& =\sum_{j \geq 1} \sum_{i \geq 1}\left|x_{i j} y_{i j}\right| \\
& =\sum_{i \geq 1} \sum_{j \geq 1}\left|x_{i j} y_{i j}\right|<\infty
\end{aligned}
$$


Sehingga, $X \in \Lambda^{\beta}$.

Proposisi 3.2 $\Lambda^{\beta} \subset \Lambda^{\gamma}$

Bukti: Diambil sebarang $X \in \Lambda^{\beta}$ maka

$$
\sum_{j \geq 1}\left|\sum_{i \geq 1} x_{i j} y_{i j}\right|<\infty \text { untuk setiap } Y \in \Lambda \text {. }
$$

Dengan kata lain, barisan $\left\{\sum_{j \geq 1}\left|\sum_{i=1}^{n} x_{i j} y_{i j}\right|\right\}$ konvergen (ke suatu bilangan kompleks).

Karena konvergen, maka barisan $\left\{\sum_{j \geq 1}\left|\sum_{i=1}^{n} x_{i j} y_{i j}\right|\right\}$ merupakan barisan Cauchy.

Selanjutnya, karena barisan Cauchy, maka barisan $\left\{\sum_{j \geq 1}\left|\sum_{i=1}^{n} x_{i j} y_{i j}\right|\right\}$ terbatas.

Dengan kata lain, untuk setiap $n$ berlaku $\sum_{j \geq 1}\left|\sum_{i=1}^{n} x_{i j} y_{i j}\right|<\infty$.

Dengan demikian diperoleh,

$$
\sup _{n} \sum_{j \geq 1}\left|\sum_{i=1}^{n} x_{i j} y_{i j}\right|<\infty \text {. }
$$

Sehingga, $X \in \Lambda^{\gamma}$.

Proposisi 3.3 $\Lambda^{\delta} \subset \Lambda^{\alpha}$

Bukti: Diambil sebarang $X \in \Lambda^{\delta}$ maka

$$
\sum_{i \geq 1} \sum_{j \geq 1}\left|x_{i j} y_{\rho(i) j}\right|<\infty \text { untuk setiap } Y \in \Lambda \text { dan setiap permutasi } \rho
$$

$\in \pi$.

Karena $\rho \in \pi$ maka dapat dipilih $\rho=\mathrm{N}$ sehingga diperoleh $\rho(i)=i$ dan

$$
\sum_{i \geq 1} \sum_{j \geq 1}\left|x_{i j} y_{i j}\right|<\infty
$$

Sehingga, $X \in \Lambda^{\alpha}$.

Proposisi 3.4 Jika $\Lambda \subset M$ maka $M^{\zeta} \subset \Lambda^{\zeta}$, dengan $\zeta=\alpha, \beta$, $\gamma$, atau $\delta$

Bukti: Misal $\Lambda \subset \mathrm{M}$. Diambil sebarang $X \in \mathrm{M}^{\alpha}$.

Untuk sebarang $Y \in \Lambda$ maka $Y \in \mathrm{M}$. Lalu karena $Y \in \mathrm{M}$ sedang $X \in \mathrm{M}^{\alpha}$ maka berlaku

$$
\sum_{i \geq 1} \sum_{j \geq 1}\left|x_{i j} y_{i j}\right|<\infty \text { di mana } x_{i j} \text { dan } y_{i j} \text { berturut-turut adalah entri }
$$
suku ke- $j$ dari barisan ke- $i$ dari $X$ dan $Y$. 
Jadi, untuk sebarang $Y \in \Lambda$ berlaku $\sum_{i \geq 1} \sum_{j \geq 1}\left|x_{i j} y_{i j}\right|<\infty$. Ini artinya bahwa $X \in \Lambda^{\alpha}$.

Jadi, $\mathrm{M}^{\alpha} \subset \Lambda^{\alpha}$.

Untuk dual- $\beta$, dual- $\gamma$, dan dual- $\delta$, bukti serupa di atas.

Proposisi 3.5 Jika $\Lambda=\Lambda_{1} \cup \Lambda_{2}$ maka $\Lambda^{\zeta}=\Lambda_{1}^{\zeta} \cap \Lambda_{2}^{\zeta}$ dengan $\zeta=\alpha, \beta$, $\gamma$, atau $\delta$

Bukti: Dimisalkan bahwa $\Lambda=\Lambda_{1} \cup \Lambda_{2}$ diperoleh $\Lambda_{1}, \Lambda_{2} \subset \Lambda$.

Karena $\Lambda_{1} \subset \Lambda$ maka $\Lambda^{\alpha} \subset \Lambda_{1}^{\alpha}$, juga karena $\Lambda_{2} \subset \Lambda$ maka $\Lambda^{\alpha} \subset \Lambda_{2}^{\alpha}$.

Dengan demikian, diperoleh $\Lambda^{\alpha} \subset \Lambda_{1}^{\alpha} \cap \Lambda_{2}^{\alpha} \ldots$ (i)

Di lain pihak, jika diambil sebarang $X \in \Lambda_{1}{ }^{\alpha} \cap \Lambda_{2}{ }^{\alpha}$ maka $X \in \Lambda_{1}{ }^{\alpha}$ dan $X$ $\in \Lambda_{2}{ }^{\alpha}$. Dengan demikian,

$$
\begin{aligned}
& \sum_{i \geq 1} \sum_{j \geq 1}\left|x_{i j} y_{i j}\right|<\infty \text { untuk setiap } Y \in \Lambda_{1} \ldots \text { (i) dan } \\
& \sum_{i \geq 1} \sum_{j \geq 1}\left|x_{i j} y_{i j}\right|<\infty \text { untuk setiap } Y \in \Lambda_{2} \ldots \ldots \text { (ii) }
\end{aligned}
$$

Sekarang, diambil sebarang $H \in \Lambda=\Lambda_{1} \cup \Lambda_{2}$ maka $H \in \Lambda_{1}$ atau $H \in \Lambda_{2}$

Jika $H \in \Lambda_{1}$ maka berdasarkan (i) diperoleh $\sum_{i \geq 1} \sum_{j \geq 1}\left|x_{i j} h_{i j}\right|<\infty$.

Jika $H \in \Lambda_{2}$ maka berdasarkan (ii) diperoleh $\sum_{i \geq 1} \sum_{j \geq 1}\left|x_{i j} h_{i j}\right|<\infty$.

Karena berlaku untuk sebarang $H \in \Lambda$ maka $X \in \Lambda^{\alpha}$.

Dengan demikian, $\Lambda_{1}{ }^{\alpha} \cap \Lambda_{2}{ }^{\alpha} \subset \Lambda^{\alpha} \quad \ldots$ (ii)

Dari (i) dan (ii) maka $\Lambda^{\alpha}=\Lambda_{1}^{\alpha} \cap \Lambda_{2}^{\alpha}$.

Untuk dual- $\beta$, dual- $\gamma$, dan dual- $\delta$, bukti serupa di atas.

Proposisi 3.6 Jika $\Lambda=\bigcup_{i=1}^{n} \Lambda_{i}$ maka $\Lambda^{\zeta}=\bigcap_{i=1}^{n} \Lambda_{i}^{\zeta}$ dengan $\zeta=\alpha, \beta, \gamma$, atau $\delta$

Bukti: Untuk $n=1$ maka benar bahwa $\Lambda=\Lambda$ maka $\Lambda^{\zeta}=\Lambda^{\zeta}$.

Misalkan pernyataan benar untuk $n=k$ yaitu

$$
\Lambda=\bigcup_{i=1}^{k} \Lambda_{i} \text { maka } \Lambda^{\zeta}=\bigcap_{i=1}^{k} \Lambda_{i}^{\zeta}
$$

Selanjutnya akan dibuktikan bahwa pernyataan benar untuk $n=k+1$.

$$
\Lambda=\bigcup_{i=1}^{k+1} \Lambda_{i}=\left\{\bigcup_{i=1}^{k} \Lambda_{i}\right\} \cup \Lambda_{k+1}
$$




$$
\begin{aligned}
\text { maka } & \Lambda^{\zeta}=\left\{\bigcup_{i=1}^{k} \Lambda_{i}\right\}^{\zeta} \cap \Lambda_{k+1}^{\zeta} \\
= & \left\{\bigcap_{i=1}^{k} \Lambda_{i}^{\zeta}\right\} \cap \Lambda_{k+1}^{\zeta} \\
= & \bigcap_{i=1}^{k+1} \Lambda_{i}^{\zeta}
\end{aligned}
$$

Jadi, benar bahwa jika $\Lambda=\bigcup_{i=1}^{n} \Lambda_{i}$ maka $\Lambda^{\zeta}=\bigcap_{i=1}^{n} \Lambda_{i}^{\zeta}$

Proposisi 3.7 $\Lambda \subset \Lambda^{\zeta \zeta}$ dengan $\zeta=\alpha, \beta, \gamma$, atau $\delta$

Bukti: Dipandang dual- $\alpha$ berikut.

$$
\Lambda^{\alpha}=\left\{X: X \in \Omega, \quad \sum_{i \geq 1} \sum_{j \geq 1}\left|x_{i j} y_{i j}\right|<\infty, \forall Y \in \Lambda\right\}
$$

Lalu, jika diambil sebarang $Y \in \Lambda$ maka $\sum_{i \geq 1} \sum_{j \geq 1}\left|x_{i j} y_{i j}\right|<\infty, \forall X \in \Lambda^{\alpha}$

Pernyataan terakhir menyatakan bahwa $Y \in\left(\Lambda^{\alpha}\right)^{\alpha}$

Jadi, $\Lambda \subset \Lambda^{\alpha \alpha}$

Untuk dual- $\beta$, dual- $\gamma$, dan dual- $\delta$, bukti serupa di atas.

Secara umum tidak berlaku $\Lambda=\Lambda^{\zeta \zeta}$. Untuk ruang barisan $\Lambda$ yang memenuhi kesamaan $\Lambda=\Lambda^{\zeta \zeta}$ maka $\Lambda$ disebut ruang sempurna (perfect space).

Proposisi 3.8 $\Lambda^{\zeta}=\Lambda^{\zeta \zeta \zeta}$ dengan $\zeta=\alpha, \beta, \gamma$, atau $\delta$

Bukti: Untuk setiap $\Lambda \subset \Omega$ berlaku $\Lambda \subset \Lambda \zeta \zeta$

Oleh karena itu, untuk ruang barisan $\Lambda^{\zeta}$ berlaku $\Lambda^{\zeta} \subset\left(\Lambda^{\zeta}\right)^{\zeta \zeta}$ atau $\Lambda^{\zeta} \subset$

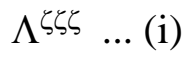

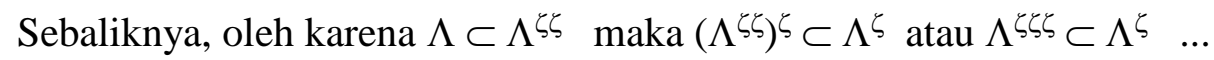
(ii)

Dari (i) dan (ii) maka $\Lambda^{\zeta}=\Lambda^{\zeta \zeta \zeta}$.

Beberapa akibat langsung dari proposisi di atas antara lain sebagai berikut.

Proposisi 3.9 Dengan $\zeta=\alpha, \beta, \gamma$, atau $\delta$ maka

(1) Untuk setiap $\Lambda \subset \Omega$ maka $\Lambda^{\zeta}$ merupakan ruang sempurna.

(2) Ruang barisan $\Lambda$ merupakan ruang sempurna jika terdapat ruang barisan $\mathrm{M} \subset \Omega$ sedemikian hingga $\mathrm{M}^{\zeta}=\Lambda$

(3) Jika $\Lambda=M^{\zeta}$ dan $M$ ruang sempurna maka $M=\Lambda^{\zeta}$. 


\section{DAFTAR PUSTAKA}

Chillingworth, H.R. (1958). Generalized "dual" sequence spaces. Nederl. Akad. Wetensch. Indag. Math. 20

Choudhary, B, dan Mishra, S.K. (1993). On Kothe-Toeplitz Duals of Certain Sequence Spaces and Their Matrix Transformations. Indian Jurnal Pure apllied Math, 24(5): 291-301

Conway, J. B. (1990). A Course in Functional Analysis. Springer-Verlag.

Garling, D.J.H. (1967). The $\beta$ - and $\gamma$-duality. Proc. Cambridge Philos. Soc. 63

Kamthan, P.K. dan Gupta, M. (1981). Sequence spaces and series. New York. Marcel Dekker, Inc.

Köthe, G. \& Toeplitz. (1934). Lineare Räume mit unendlich vielen Koordinaten und Ringe unendlicher Matrizen, Jour. Reine angew. Math. 171

Royden, H. L. (1988). Real Analysis. MacMillan Publishing Company. New York.

Rudin, W. (1973). Functional analysis. New York: McGraw-Hill Book Company

Wilansky, A. (1984). Summability through Fuctional Analysis. Amsterdam: North Holland. 